Bull. Korean Math. Soc. 52 (2015), No. 1, pp. 215-222

http://dx.doi.org/10.4134/BKMS.2015.52.1.215

\title{
HOMOLOGY OF CONTACT CR-WARPED PRODUCT SUBMANIFOLDS OF AN ODD-DIMENSIONAL UNIT SPHERE
}

\author{
Bayram ŞAHIN AND Fulya ŞAhin
}

\begin{abstract}
We show that homology group on a contact CR-warped product submanifold in odd dimensional sphere is zero under certain conditions in terms of warping function and the dimension of the submanifold.
\end{abstract}

\section{Introduction}

The homology groups of a manifold are important topological invariants that provide an algebraic summary of the manifold. Among other things, these groups contain rich topological information about the connected components, holes, tunnels and dimension of the manifold and this theory has many applications. Indeed, homology theory has its applications in gene expression data, protein docking, image segmentation and root architecture [8].

There is a close relationship between homology theory and submanifold theory. In this direction we note that Federer and Fleming [9] showed that any non-trivial integral homology class in $H_{p}(M, \mathbb{Z})$ ) corresponds to a stable current. Later Lawson and Simons [15] showed that there are no stable integral currents in the sphere $S^{n}$, and there is no integral current in a submanifold $M^{m}$ of $S^{n}$ when the second fundamental form of $M^{m}$ satisfies a pinching condition. The result on submanifolds of $S^{n}$ has been extended to Euclidean space by Leung [16] and Xin [19]. It has been also extended to $S^{n_{1}} \times S^{n_{2}}$ by Zhang [21]. Recently Liu and Zhang obtain the non-existence theorems of stable integral currents for certain classes of hypersurfaces or higher codimensional submanifolds in the Euclidean spaces [17].

On the other hand, Chen defined CR-warped product submanifolds of almost Hermitian manifolds by using the notion of CR-submanifolds [1] and warped product manifolds [2] in his series papers [4], [5], [6]. The notion of contact CRwarped product submanifolds of a Sasakian manifold was defined by Hasegawa

Received December 17, 2013; Revised March 7, 2014.

2010 Mathematics Subject Classification. 55N25, 53C65, 53C40, 53C15.

Key words and phrases. contact CR-warped submanifold, stable current, homology group. 
and Mihai in [10] and they showed that the only warped products with nonconstant warping function which are contact CR-submanifolds in a Sasakian manifold $\bar{M}$ have the form $M=M_{T} \times{ }_{f} M_{\perp}$ with $M_{T}$ an invariant submanifold tangent to the characteristic vector field $\xi$ and $M_{\perp}$ a $C$-totally real submanifold of $\bar{M}$. They simply called such submanifolds contact CR-warped products. CRwarped product submanifolds have been studied by many authors in different ambient spaces, see a survey paper [7] and references therein.

Contact CR-submanifolds of an odd dimensional sphere have nice geometric properties and such submanifolds have been studied widely (see [11], [12], [13], [14], [18]). In this paper, we obtain an inequality in terms of second fundamental form of the submanifold and warping function which gives information on the homology group of contact CR-warped product submanifolds in an odd dimensional sphere.

\section{Preliminaries}

An odd dimensional Riemannian manifold $(\bar{M}, g)$ is called a contact metric manifold [3] if there is a $(1,1)$ tensor field $\phi$, a vector field $\xi$, called the characteristic vector field and its 1 -form $\eta$ such that

$$
\begin{aligned}
g(\phi X, \phi Y) & =g(X, Y)-\eta(X) \eta(Y), \eta(\xi=1, \\
\phi^{2}(X) & =-X+\eta(X) \xi, \quad g(X, \xi)=\eta(X), \\
d \eta(X, Y) & =g(X, \phi Y), \quad \forall X, Y \in \Gamma(T M) .
\end{aligned}
$$

It follows that $\phi \xi=0, \eta \circ \phi=0$. Then $(\phi, \xi, \eta, g)$ is called contact metric structure of $\bar{M} \cdot \bar{M}$ has a normal contact structure if $N_{\phi}+d \eta \otimes \xi=0$, where $N_{\phi}$ is the Nijenhuis tensor field of $\phi[20]$. A normal contact metric manifold is called a Sasakian manifold [3] for which we have

$$
\begin{aligned}
\bar{\nabla}_{X} \xi & =\phi X, \\
\left(\bar{\nabla}_{X} \phi\right) Y & =-g(X, Y) \xi+\eta(Y) X .
\end{aligned}
$$

A plane section $P$ in $T_{x} \bar{M}$ of a Sasakian manifold $\bar{M}$ is called a $\phi$-section if it is spanned by a unit vector $X$ orthogonal to $\xi$ and $\phi X$, where $X$ is a non-null vector field on $\bar{M}$. The sectional curvature $K(P)$ with respect to $P$ determined by $X$ is called a $\phi$-sectional curvature. If $\bar{M}$ has a $\phi$-sectional curvature $c$ which does not depend on the $\phi$-section at each point, then $M$ is constant in $\bar{M}$. Then, $\bar{M}$ is called a Sasakian space form, denoted by $\bar{M}(c)$. The curvature tensor $\bar{R}$ of a Sasakian space form $\bar{M}(c)$ is given by [3]

$$
\begin{aligned}
\bar{R}(X, Y) Z= & \frac{(c+3)}{4}\{g(Y, Z) X-g(X, Z) Y\}+\frac{(c-1)}{4}\{\eta(X) \eta(Z) Y \\
& -\eta(Y) \eta(Z) X+g(X, Z) \eta(Y) \xi-g(Y, Z) \eta(X) \xi \\
& +g(\phi Y, Z) \phi X+g(\phi Z, X) \phi Y-2 g(\phi X, Y) \phi Z\}
\end{aligned}
$$

for any $X, Y$ and $Z$ vector fields on $\bar{M}$. 
Let $M$ be a Riemannian manifold isometrically immersed in $\bar{M}$ and denote by the same symbol $g$ for the Riemannian metric induced on $M$. Let $\Gamma(T M)$ be the Lie algebra of vector fields in $M$ and $\Gamma\left(T M^{\perp}\right)$ the set of all vector fields normal to $M$, same notation for smooth sections of any other vector bundle $E$. Denote by $\nabla$ the Levi-Civita connection of $M$. Then the Gauss and Weingarten formulas are given by

$$
\bar{\nabla}_{X} Y=\nabla_{X} Y+h(X, Y)
$$

and

$$
\bar{\nabla}_{X} N=-A_{N} X+\nabla_{X}^{\perp} N
$$

for any $X, Y \in \Gamma(T M)$ and any $N \in \Gamma\left(T M^{\perp}\right)$, where $\nabla^{\perp}$ is the connection in the normal bundle $T M^{\perp}, h$ is the second fundamental form of $M$ and $A_{N}$ is the Weingarten endomorphism associated with $N$. The second fundamental form $h$ and the shape operator $A$ are related by

$$
g\left(A_{N} X, Y\right)=g(h(X, Y), N)
$$

Let $R$ be the Riemann curvature tensor of $M$. Then the equation of Gauss is given by

$$
\begin{aligned}
g(\bar{R}(X, Y) Z, W)= & g(R(X, Y) Z, W)-g(h(X, W), h(Y, Z)) \\
& +g(h(Y, W), h(X, Z))
\end{aligned}
$$

for $X, Y, Z, W \in \Gamma(T M)$.

Let $\left(B, g_{1}\right)$ and $\left(F, g_{2}\right)$ be two Riemannian manifolds, $f: B \rightarrow(0, \infty)$ and $\pi: B \times F \rightarrow B, \eta: B \times F \rightarrow F$ the projection maps given by $\pi(p, q)=p$ and $\eta(p, q)=q$ for every $(p, q) \in B \times F$. The warped product ([2]) $M=B \times_{f} F$ is the manifold $B \times F$ equipped with the Riemannian structure such that

$$
g(X, Y)=g_{1}\left(\pi_{*} X, \pi_{*} Y\right)+(f \circ \pi)^{2} g_{2}\left(\eta_{*} X, \eta_{*} Y\right)
$$

for every $X$ and $Y$ of $M$, where $*$ denotes the differential map. The function $f$ is called the warping function of the warped product manifold. In particular, if the warping function is constant, then the warped product manifold $M$ is said to be trivial. Let $X, Y$ be vector fields on $B$ and $V, W$ vector fields on $F$, then from Lemma 7.3 of [2], we have

$$
\nabla_{X} V=\nabla_{V} X=\left(\frac{X f}{f}\right) V
$$

where $\nabla$ is the Levi-Civita connection on $M$. Since $[X, V]=$, by using (8) we have

$$
R(X, V) Y=\frac{H^{f}(X, Y)}{f} V
$$

for $X, Y \in \Gamma\left(T B_{1}\right)$ and $V \in \Gamma(T F)$, where $H^{f}$ is the Hessian of $f$.

A submanifold $M$ normal to $\xi$ in a Sasakian manifold is said to be $C$-totally real submanifold. In this case $\phi$ maps any tangent space of $M$ into the normal space, that is, $\phi\left(T_{x} M\right) \subset T_{x} M^{\perp}$ for every $x \in M$. For submanifolds tangent to 
the structure vector field $\xi$, there are different classes of submanifolds. A submanifold $M$ tangent to $\xi$ is called an invariant submanifold if $\phi\left(T_{x} M\right) \subset T_{x} M$, for every $x \in M$, where $T_{x} M$ denotes the tangent space of $M$ at the point $x$. $M$ is called an anti-invariant submanifold if $\phi\left(T_{x} M\right) \subset T_{x} M^{\perp}$ for every $x \in M$, where $T_{x} M^{\perp}$ denotes the normal space of $M$ at the point $x$. The submanifold $M$ is called a contact CR-submanifold if there exists a differentiable distribution $D: x \rightarrow D_{x} \subset T_{x} M$ such that $D$ is invariant with respect to $\phi$ and the complementary distribution $D^{\perp}$ is anti-invariant with respect to $\phi$. A submanifold $M$ of a Sasakian manifold $\bar{M}$ is called contact CR-warped product [10] if it is the warped product $M_{T} \times_{f} M_{\perp}$ of an invariant submanifold $M_{T}$ tangent to $\xi$ and a $C$-totally real submanifold $M_{\perp}$ of $\bar{M}$. For a contact CR-warped product submanifold of a Sasakian manifold, Hasegawa and Mihai proved the following formula.

Lemma 2.1 ([10]). For a contact $C R$-warped product $M=M_{T} \times_{f} M_{\perp}$ in any Sasakian manifold $\bar{M}$, we have

$$
g(h(\phi X, Z), \phi V)=X(\ln f) g(Z, V)
$$

and

$$
h(\xi, Z)=\phi Z
$$

for $X \in \Gamma(D)$ and $Z, V \in \Gamma\left(D^{\perp}\right)$.

\section{Homology of contact CR-warped submanifolds}

In this section, we are going to show that there are no stable currents on contact CR-warped product submanifolds under certain conditions. We first recall the following result which combines the results of Lawson-Simons, Leung and Xin.

Lemma 3.1 ([9], [16], [19]). Let $M^{n}$ be a compact, $n$-dimensional submanifold of the space form $\bar{M}(c)$ of curvature $c \geq 0$ with second fundamental form $h$, and let $p, q$ be positive integers such that $p+q=n$. If for any $x \in M^{n}$ and any orthonormal basis $\left\{e_{1}, \ldots, e_{n}\right\}$ of the tangent space $T_{x} M^{n}$, the inequality

$$
\sum_{i=1}^{p} \sum_{s=p+1}^{n}\left(2\left|h\left(e_{i}, e_{s}\right)\right|^{2}-g\left(h\left(e_{i}, e_{i}\right), h\left(e_{s}, e_{s}\right)\right)\right)<p q c
$$

is satisfied, then there are no stable $p$ currents in $M^{n}$. Moreover, $H_{p}\left(M^{n}, \mathbb{Z}\right)=$ $0, H_{q}\left(M^{n}, \mathbb{Z}\right)=0$, where $H_{i}(M, \mathbb{Z})$ is the $i$-th homology group of $M$ with integer coefficients.

Let $S^{2 m+1}$ be a $(2 m+1)$-sphere. For any point $z \in S^{2 m+1}$, we put $J z=\xi$, where $J$ denotes the complex structure of the complex $m+1$ space $\mathbb{C}^{m+1}$. We consider the orthogonal projection $\pi: T_{z} \mathbb{C}^{m+1} \longrightarrow T_{z} S^{2 m+1}$. Putting $\phi=\pi \circ J$, one can see that $(\phi, \xi, \eta, g)$ is a Sasakian structure on $S^{2 m+1}$, where $\eta$ is a 1 -form dual to $\xi$ and $g$ the standard metric tensor field on $S^{2 m+1}$. 
Thus $S^{2 m+1}$ can be considered as a Sasakian manifold of constant $\phi$ sectional curvature 1 , that is, of constant curvature 1 (cf. [20]).

Theorem 3.1. Let $M^{p+q+1}=M_{T}^{p+1} \times_{f} M_{\perp}^{q}$ be a compact contact CR-warped product submanifold of $S^{2\left(\frac{p}{2}+q\right)+1}$. If

$$
\Delta f>\frac{1}{f}\|\nabla f\|_{D-s p\{\xi\}}^{2}+f-\frac{q}{f}\|\nabla f\|^{2},
$$

then there are no stable $p+1$ currents in $M^{p+1+q}$. Moreover, $H_{p+1}\left(M^{n}, \mathbb{Z}\right)=0$, $H_{q}\left(M^{n}, \mathbb{Z}\right)=0$, where $H_{i}(M, \mathbb{Z})$ is the $i$-th homology group of $M$ with integer coefficients and $\nabla f$ is the gradient of $f$.

Proof. Let $\left\{\xi, e_{1}, \ldots, e_{p}, n_{1}, \ldots, n_{q}\right\}$ be a local orthonormal frame of $M$ such that $\left\{\xi, e_{1}, \ldots, e_{p}\right\}$ is an orthonormal frame of $M_{T}$ and $\left\{n_{1}, \ldots, n_{q}\right\}$ is an orthonormal frame of $M_{\perp}$. Then (7) and the Sasakian manifold $S^{2\left(\frac{p}{2}+q\right)+1}$ of constant curvature 1 imply that

$$
\begin{aligned}
& \sum_{i=1}^{p+1} \sum_{k=1}^{q} g\left(R\left(e_{i}, n_{k}\right) e_{i}, n_{k}\right) \\
= & -(p+1) q+\sum_{i=1}^{p+1} \sum_{k=1}^{q}-g\left(h\left(n_{k}, n_{k}\right), h\left(e_{i}, e_{i}\right)\right)+\left\|h\left(e_{i}, n_{k}\right)\right\|^{2} .
\end{aligned}
$$

Hence we get

$$
\begin{aligned}
& \sum_{i=1}^{p+1} \sum_{k=1}^{q} g\left(R\left(e_{i}, n_{k}\right) e_{i}, n_{k}\right)+\left\|h\left(e_{i}, n_{k}\right)\right\|^{2} \\
= & -(p+1) q+\sum_{i=1}^{p+1} \sum_{k=1}^{q}-g\left(h\left(n_{k}, n_{k}\right), h\left(e_{i}, e_{i}\right)\right)+2\left\|h\left(e_{i}, n_{k}\right)\right\|^{2} .
\end{aligned}
$$

On the other hand, from (9), we have

$$
R\left(e_{i}, n_{k}\right) e_{i}=\frac{H^{f}\left(e_{i}, e_{i}\right)}{f} n_{k},
$$

where $H^{f}$ is the Hessian form of $f$. Thus we derive

$$
\sum_{i=1}^{p+1} \sum_{k=1}^{q} g\left(R\left(e_{i}, n_{k}\right) e_{i}, n_{k}\right)=\frac{q}{f} \sum_{i=1}^{p+1} g\left(\nabla_{e_{i}} \nabla f, e_{i}\right) .
$$

Putting (16) in (14) we get

$$
\begin{aligned}
& \sum_{i=1}^{p+1}\left(\frac{q}{f} g\left(\nabla_{e_{i}} \nabla f, e_{i}\right)+\sum_{k=1}^{q}\left\|h\left(e_{i}, n_{k}\right)\right\|^{2}\right) \\
= & -(p+1) q+\sum_{i=1}^{p+1} \sum_{k=1}^{q} 2\left\|h\left(e_{i}, n_{k}\right)\right\|^{2}-g\left(h\left(n_{k}, n_{k}\right), h\left(e_{i}, e_{i}\right)\right) .
\end{aligned}
$$


Since

$$
\Delta f=-\operatorname{div} \nabla f=-\sum_{i=1}^{p+1} g\left(\nabla_{e_{i}} \nabla f, e_{i}\right)-\sum_{k=1}^{q} g\left(\nabla_{n_{k}} \nabla f, n_{k}\right),
$$

using (8) we derive

$$
\Delta f=-\sum_{i=1}^{p+1} g\left(\nabla_{e_{i}} \nabla f, e_{i}\right)-\frac{q}{f}\|\nabla f\|^{2} .
$$

Using (17) in (18) we arrive at

$$
\begin{aligned}
& \Delta f-\frac{f}{q} \sum_{i=1}^{p+1} \sum_{k=1}^{q}\left\|h\left(e_{i}, n_{k}\right)\right\|^{2} \\
= & f(p+1)-\frac{f}{q} \sum_{i=1}^{p+1} \sum_{k=1}^{q}\left\{2\left\|h\left(e_{i}, n_{k}\right)\right\|^{2}-g\left(h\left(n_{k}, n_{k}\right), h\left(e_{i}, e_{i}\right)\right)\right\}-\frac{q}{f}\|\nabla f\|^{2} .
\end{aligned}
$$

Moreover, if $\left\{n_{1}, \ldots, n_{k}\right\}$ is an orthonormal frame of $M_{\perp}$, then $\left\{\phi n_{1}, \ldots, \phi n_{k}\right\}$ is the orthonormal frame of $T M^{\perp}$ since $\phi\left(D^{\perp}\right)=T M^{\perp}$. Thus we write

$$
\left\|h\left(e_{i}, n_{k}\right)\right\|^{2}=\sum_{i=1}^{p+1} \sum_{k=1}^{q} g\left(h\left(e_{i}, n_{k}\right), \phi n_{k}\right)^{2} .
$$

Then using (10) and (11) we obtain

$$
\left\|h\left(e_{i}, n_{k}\right)\right\|^{2}=\|\nabla(\ln f)\|_{D-s p\{\xi\}}^{2} q+q .
$$

Hence we have

$$
\left\|h\left(e_{i}, n_{k}\right)\right\|^{2}=\frac{q}{f^{2}}\|\nabla f\|_{D-s p\{\xi\}}^{2}+q .
$$

Using (20) in (19) we derive

$$
\begin{aligned}
& -\frac{q}{f} \Delta f+\frac{q}{f^{2}}\|\nabla f\|_{D-s p\{\xi\}}^{2}+q(p+2)-\frac{q^{2}}{f^{2}}\|\nabla f\|^{2} \\
= & \sum_{i=1}^{p+1} \sum_{k=1}^{q}\left\{2\left\|h\left(e_{i}, n_{k}\right)\right\|^{2}-g\left(h\left(n_{k}, n_{k}\right), h\left(e_{i}, e_{i}\right)\right)\right\} .
\end{aligned}
$$

Then proof follows from (21) and Lemma 3.1.

We also have the following result.

Corollary 3.1. Let $M^{2 p+q+1}=M_{T}^{2 p+1} \times_{f} M_{\perp}^{q}$ be a compact contact $C R$-warped product submanifold of $S^{2(p+q)+1}$. If

$$
\|\nabla f\|_{D-s p\{\xi\}}^{2}<\frac{f^{2}(2 p-1)}{2},
$$


then there are no stable $2 p+1$ currents in $M^{2 p+1+q}$. Moreover, $H_{2 p+1}\left(M^{n}, \mathbb{Z}\right)=$ $0, H_{q}\left(M^{n}, \mathbb{Z}\right)=0$, where $H_{i}(M, \mathbb{Z})$ is the $i$-th homology group of $M$ with integer coefficients and $\nabla f$ is the gradient of $f$.

Proof. We first show that $g(h(X, Y), \phi Z)=0$ for $X, Y \in \Gamma(D)$ and $Z \in \Gamma\left(D^{\perp}\right)$. Indeed, from (4) we get

$$
g(h(X, Y), \phi Z)=g\left(-\phi \bar{\nabla}_{X} Y, Z\right) .
$$

Hence we have

$$
g(h(X, Y), \phi Z)=g\left(-\bar{\nabla}_{X} \phi Y+\left(\bar{\nabla}_{X} \phi\right) Y, Z\right) .
$$

Then from (2) and (4) we obtain

$$
g(h(X, Y), \phi Z)=g\left(-\nabla_{X} \phi Y, Z\right)=g\left(\phi Y, \nabla_{X} Z\right) .
$$

Using (8) we derive

$$
g(h(X, Y), \phi Z)=X(\ln f) g(\phi Y, Z)=0 .
$$

From this, we have

$$
\sum_{i=1}^{2 p+1} \sum_{k=1}^{q}\left\{2\left\|h\left(e_{i}, n_{k}\right)\right\|^{2}-g\left(h\left(n_{k}, n_{k}\right), h\left(e_{i}, e_{i}\right)\right)\right\}=\sum_{i=1}^{2 p+1} \sum_{k=1}^{q} 2\left\|h\left(e_{i}, n_{k}\right)\right\|^{2} .
$$

Using (20) we obtain

$$
\sum_{i=1}^{2 p+1} \sum_{k=1}^{q}\left\{2\left\|h\left(e_{i}, n_{k}\right)\right\|^{2}-g\left(h\left(n_{k}, n_{k}\right), h\left(e_{i}, e_{i}\right)\right)\right\}=\frac{2 q}{f^{2}}\|\nabla f\|_{D-s p\{\xi\}}^{2}+2 q .
$$

Then proof follows from Lemma 3.1.

\section{References}

[1] A. Bejancu, Geometry of CR-Submanifolds, Kluwer Academic Publishers, Dortrecht, 1986.

[2] R. L. Bishop and B. O'Neill, Manifolds of negative curvature, Trans. Amer. Math. Soc. 145 (1969), 1-49.

[3] D. E. Blair, Riemannian geometry of contact and symplectic manifolds, Progress in Mathematics, 203. Birkhäuser, 2002.

[4] B. Y. Chen, Geometry of warped product CR-submanifolds in Kaehler manifold, Monatsh. Math. 133 (2001), no. 3, 177-195.

[5] — Geometry of warped product CR-submanifolds in Kaehler manifolds II, Monatsh. Math. 134 (2001), no. 2, 103-119.

[6] — CR-warped products in complex projective spaces with compact holomorphic factor, Monatsh. Math. 141 (2004), no. 3, 177-186.

[7] — Geometry of warped product submanifolds: A survey, J. Adv. Math. Stud. 6 (2013), no. 2, 1-43.

[8] H. Edelsbrunner and J. Harer, Computational Topology: An Introduction, Lecture Notes, Duke University, 2013.

[9] H. Federer and W. Fleming, Normal and integral currents, Ann. of Math. 72 (1960), 458-520.

[10] I. Hasegawa and I. Mihai, Contact CR-warped product submanifolds in Sasakian manifolds, Geom. Dedicata 102 (2003), 143-150. 
[11] H. S. Kim and J. S. Pak, Sectional curvature of contact CR-submanifolds of an odddimensional unit sphere, Bull. Korean Math. Soc. 42 (2005), no. 4, 777-787.

[12] _ Certain contact CR-submanifolds of an odd-dimensional unit sphere, Bull. Korean Math. Soc. 44 (2007), no. 1, 109-116.

[13] - Certain class of contact CR-submanifolds of an odd-dimensional unit sphere, Taiwanese J. Math. 14 (2010), no. 2, 629-646.

[14] _ Scalar curvature of contact CR-submanifolds in an odd-dimensional unit sphere, Bull. Korean Math. Soc. 47 (2010), no. 3, 541-549.

[15] H. B. Lawson and J. Simons, On stable currents and their application to global problems in real and complex geometry, Ann. of Math. 98 (1973), 427-450.

[16] P. F. Leung, On a relation between the topology and the intrinsic and extrinsic geometries of a compact submanifold, Proc. Edinburgh Math. Soc. 28 (1985), no. 3, 305-311.

[17] J. Liu and Q. Zhang, Non-existence of stable currents in submanifolds of the Euclidean spaces, J. Geom. 96 (2009), no. 1-2, 125-133.

[18] J. S. Pak, J.-H. Kwon, H. S. Kim, and Y.-M. Kim, Contact CR-submanifolds of an odd-dimensional unit sphere, Geom. Dedicata 114 (2005), 1-11.

[19] Y. L. Xin, An application of integral currents to the vanishing theorems, Sci. Sinica Ser. A 27 (1984), no. 3, 233-241.

[20] K. Yano and M. Kon, Structures on Manifolds, World Scientific, 1984.

[21] X. S. Zhang, Nonexistence of stable currents in submanifolds of a product of two spheres, Bull. Austral. Math. Soc. 44 (1991), no. 2, 325-336.

BAYRAM ŞAHIN

Department of Mathematics

INONU UNIVERSITY

44280, Malatya, Turkey

E-mail address: bayram.sahin@inonu.edu.tr

Fulya Şahin

Department of Mathematics

INONU UNIVERSITY

44280, Malatya, Turkey

E-mail address: fulya.sahin@inonu.edu.tr 